\title{
$\mathbb{Z}_{3}$ Polyakov Loop Models and Inverse Monte-Carlo Methods
}

\author{
Christian Wozar*, Tobias Kästner, Sebastian UhImann, Andreas Wipf \\ Theoretisch-Physikalisches Institut, Friedrich-Schiller-Universität Jena, Max-Wien-Platz 1, \\ 07743 Jena, Germany \\ E-mail: christian.wozar@uni-jena.de
}

\section{Thomas Heinzl}

School of Mathematics and Statistics, University of Plymouth, Drake Circus, Plymouth, PL4 8AA, United Kingdom

\begin{abstract}
We study effective Polyakov loop models for $S U$ (3) Yang-Mills theory at finite temperature. A comprehensive mean field analysis of the phase diagram is carried out and compared to the results obtained from Monte-Carlo simulations. We find a rich phase structure including ferromagnetic and antiferromagnetic phases. Due to the presence of a tricritical point the mean field approximation agrees very well with the numerical data. Critical exponents associated with second-order transitions coincide with those of the $\mathbb{Z}_{3}$ Potts model. Finally, we employ inverse Monte-Carlo methods to determine the effective couplings in order to match the effective models to Yang-Mills theory.
\end{abstract}

The XXV International Symposium on Lattice Field Theory

July 30-4 August 2007

Regensburg, Germany

\footnotetext{
${ }^{*}$ Presenter.
} 


\section{Introduction}

The Svetitsky-Yaffe conjecture [1, 2] states that the Yang-Mills finite temperature transition in dimension $d+1$ is described by an effective spin model in $d$ dimensions with short range interactions. Combining this idea with strong coupling expansions and inverse Monte-Carlo (IMC) methods we analyse the relationship between $S U(3)$ YM theory in $3+1$ dimensions and effective theories formulated as $\mathbb{Z}_{3}$ spin models in 3 dimensions.

\section{2. $S U(3)$ and characters of representations}

Our effective operators are class functions on $S U(3)$. With group elements in diagonal form, $g=$ $\operatorname{diag}\left(e^{i \phi_{1}}, e^{i \phi_{2}}, e^{-i\left(\phi_{1}+\phi_{2}\right)}\right)$, we associate a group character in the fundamental representation by

$$
\mathscr{P} \equiv \operatorname{tr} g \equiv \chi_{10}(g)=e^{i \phi_{1}}+e^{i \phi_{2}}+e^{-i\left(\phi_{1}+\phi_{2}\right)},
$$

with the typical example being the Polyakov loop. The parameterisation (2.1) implies the reduced Haar measure on the maximal Abelian torus,

$$
d \mu_{\mathrm{red}}=J^{2} d \phi_{1} d \phi_{2}, \quad J^{2}=15-6 \chi_{11}+3 \chi_{30}+3 \chi_{03}-\chi_{22}
$$

Using Young tableaux one can express all characters $\chi_{p q}$ with Dynkin labels $[p, q]$ in terms of the fundamental ones, $\mathscr{P}$ and $\mathscr{P}^{*}$.

\section{Observables}

We discuss YM theory on a $N_{\mathrm{s}}^{3} \times N_{\mathrm{t}}$-lattice. The Polyakov loop $\mathscr{P}_{\boldsymbol{x}}$ is measured in terms of its lattice average,

$$
P \equiv \frac{1}{V} \sum_{\boldsymbol{x}} \mathscr{P}_{\boldsymbol{x}}, \quad V=N_{\mathrm{s}}^{3}
$$

The observable relevant for the analysis of antiferromagnetic phases is

$$
M \equiv \frac{1}{V} \sum_{\boldsymbol{x}} \mathscr{P}_{\boldsymbol{x}} \operatorname{sgn}(\boldsymbol{x}), \quad \operatorname{sgn}(\boldsymbol{x}) \equiv(-1)^{\sum_{i} x_{i}}
$$

and measures the difference of the Polyakov loop on odd and even sublattices.

Since we will have to deal with phases where the traced Polyakov loop is located halfway between the $S U(3)$ center elements we project the value of the traced Polyakov loop onto the nearest $\mathbb{Z}_{3}$-axis and define a rotated Polyakov loop by

$$
P_{r}=\left\{\begin{array}{cl}
\operatorname{Re} P & : P \in \mathscr{F} \\
-\frac{1}{2} \operatorname{Re} P+\frac{\sqrt{3}}{2} \operatorname{Im} P & : P \in \mathscr{F}{ }^{\prime} \\
-\frac{1}{2} \operatorname{Re} P-\frac{\sqrt{3}}{2} \operatorname{Im} P & : P \in \mathscr{F}^{\prime \prime}
\end{array}\right.
$$

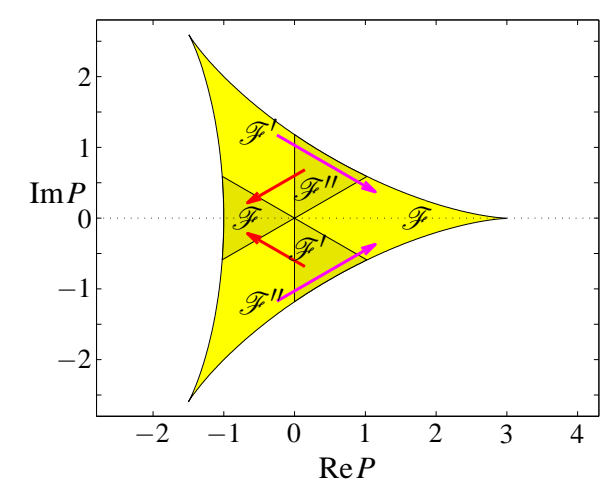




\section{Effective models for Yang-Mills theory}

We start with the well-known lattice Wilson action

$$
S_{\mathrm{W}}=\beta \sum_{\square}\left(1-\frac{1}{N_{C}} \operatorname{Re} \operatorname{tr} U_{\square}\right), \quad \beta=\frac{6}{a^{4} g^{2}}
$$

and perform a strong coupling expansion (for small $\beta$ ). Since the resulting 'operators' (Polyakov loop monomials) are dimensionless there is no natural ordering scheme. We therefore use a truncation scheme based on:

- Ordering by powers of $\beta$ which are closely related to the dimension of the corresponding group representations.

- Ordering by the distance across which the Polyakov loops are coupled.

In compact form the strong coupling expansion is given by

$$
S_{\text {eff }}=\sum_{r} \sum_{\mathscr{R}_{1} \ldots \mathscr{R}_{r} \ell_{1} \ldots \ell_{r}} c_{\mathscr{R}_{1} \ldots \mathscr{R}_{r}}^{\ell_{1} \ldots \ell_{r}}(\beta) \prod_{i=1}^{r} S_{\mathscr{R}_{i}, \ell_{i}}=\sum_{i} \lambda_{i} S_{i}
$$

with the basic building blocks

$$
S_{\mathscr{R}, \ell} \equiv \chi_{\mathscr{R}}\left(\mathscr{P}_{\boldsymbol{x}}\right) \chi_{\mathscr{R}}^{*}\left(\mathscr{P}_{\boldsymbol{y}}\right)+\text { c.c. }, \quad \ell \equiv\langle\boldsymbol{x} \boldsymbol{y}\rangle .
$$

Here $r$ counts the number of link operators contributing at each order. The coefficients $c_{\mathscr{R}_{1} \ldots \mathscr{R}_{r}}^{\ell_{1} \ldots \ell_{r}}$ are the couplings between the operators $S_{\mathscr{R}_{i}, \ell_{i}}$ sitting at nearest-neighbor (NN) links $\ell_{i} \equiv\left\langle\boldsymbol{x}_{i}, \boldsymbol{y}_{i}\right\rangle$ in representation $\mathscr{R}_{i}$. The effective action hence describes a network of link operators that are collected into (possibly disconnected) 'polymers' contributing with 'weight' $c_{\mathscr{R}_{1} \ldots \mathscr{R}_{r}}^{\ell_{1} \ldots \ell_{r}}$. One expects the 'weights' or couplings to decrease as the dimensions of the involved representations and interlink distances increase. In a strong coupling ( small $\beta$ ) expansion truncated at $\mathscr{O}\left(\beta^{k N_{\mathrm{t}}}\right)$ one has $r \leq k$ and the additional restriction $\left|\mathscr{R}_{1}\right|+\cdots+\left|\mathscr{R}_{r}\right|<k$ with $|\mathscr{R}| \equiv p+q$ for a given representation $\mathscr{R}$ with Dynkin labels $[p, q]$.

\section{A toy model - mean field vs. Monte-Carlo}

We consider the $S U(3)$ model $[3,4]$

$$
S=\lambda_{1} \sum_{\langle\boldsymbol{x} \boldsymbol{y}\rangle}\left(\chi_{10}\left(\mathscr{P}_{\boldsymbol{x}}\right) \chi_{01}\left(\mathscr{P}_{\boldsymbol{y}}\right)+\text { c.c. }\right)+\lambda_{4} \sum_{\langle\boldsymbol{x} \boldsymbol{y}\rangle}\left(\chi_{10}\left(\mathscr{P}_{\boldsymbol{x}}\right) \chi_{20}\left(\mathscr{P}_{\boldsymbol{y}}\right)+\chi_{20}\left(\mathscr{P}_{\boldsymbol{x}}\right) \chi_{10}\left(\mathscr{P}_{\boldsymbol{y}}\right)+\text { c.c. }\right) \text {. }
$$

A mean field approximation can be applied to approximately determine the associated phase diagram. We use the following ansatz for the distribution $p$ of the field $\mathscr{P}$,

$$
p[\mathscr{P}] \rightarrow p_{\mathrm{mf}}[\mathscr{P}] \equiv \prod_{\boldsymbol{x}} p_{\boldsymbol{x}}\left(\mathscr{P}_{\boldsymbol{x}}\right) \quad \text { with } \quad p_{\boldsymbol{x}}\left(\mathscr{P}_{\boldsymbol{x}}\right)= \begin{cases}p_{\mathrm{e}}\left(\mathscr{P}_{\boldsymbol{x}}\right) & : \operatorname{sgn}(\boldsymbol{x})=1 \\ p_{\mathrm{o}}\left(\mathscr{P}_{\boldsymbol{x}}\right) & : \operatorname{sgn}(\boldsymbol{x})=-1\end{cases}
$$

The resulting phase diagram is displayed in Fig. 1 (left panel).

A straightforward Monte-Carlo simulation on an $8^{3}$-lattice with a Metropolis algorithm using our jenLaTT package leads to a phase diagram (Fig. 1, right panel) similar to the one obtained by the mean field analysis. This agreement is due to the presence of a tri-critical point implying an upper critical dimension of three. In summary, the full phase structure consists of a symmetric phase (in the center of each panel of Fig. 1), a ferromagnetic phase (upper left), an anti-center phase (lower left) and an antiferromagnetic (lower right) phase. The anti-center phase is related to the 'skewed' phase of [5]. 

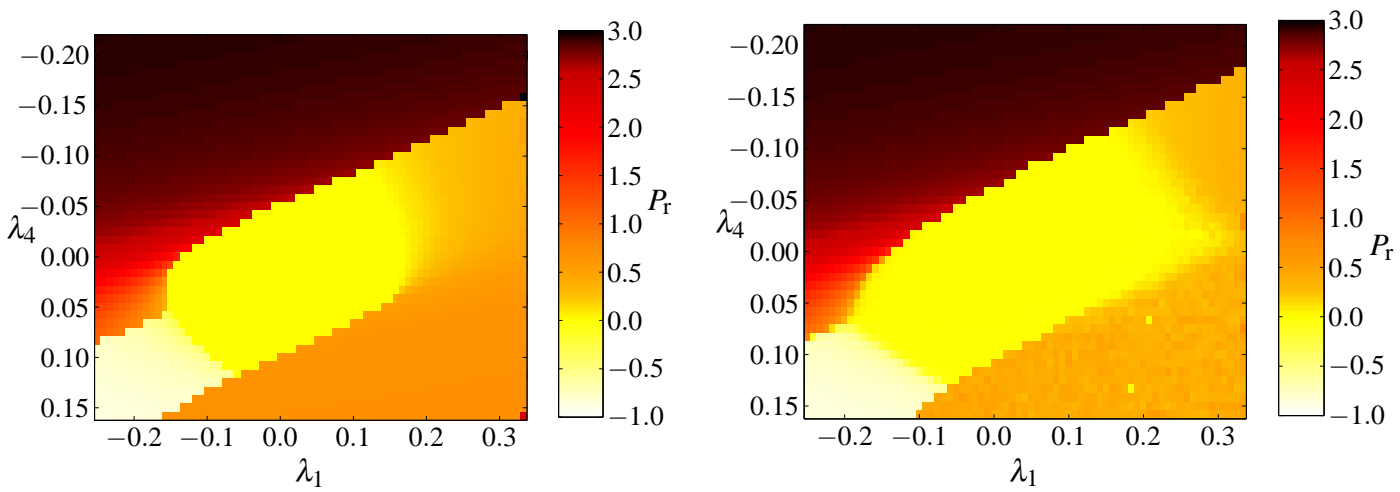

Figure 1: Phase diagrams obtained by mean field analysis (left) and Monte-Carlo simulation (right).

\section{Algorithms for first and second order transitions}

The simulations for the microscopic YM theory were done using standard heat bath algorithms. For the effective models we employed standard Metropolis updates to find the phase diagram. In the vicinity of phase transitions we made use of the following specially designed update scheme.

For first order transitions we used a multicanonical algorithm [6] improving the transition rate near critical points. For larger lattices the distribution $\rho$ of the order parameter (denoted $\ell$ ) was predicted using the scaling relation

$$
\log \rho(\ell, V) \approx A(\ell)+C(\ell) V
$$

For second order phase transitions algorithms of Wolff or Swendsen-Wang type lead to strong suppression of the dynamical critical exponent. These algorithms are useful for systems with involutory global symmetries, where the local application of such symmetries leads to ergodic behavior of the system. For our system there is no ergodic symmetry. So we had to modify the well-known Wolff cluster algorithm [7] as follows:

1. Choose a random number $N_{M}$ between 0 and $V=N^{3}$.

2. Do $N_{M}$ standard Metropolis sweeps at randomly drawn lattice points.

3. For a suitable fixed number $N_{\mathrm{cl}}$ repeat the steps for building a cluster by using the complex conjugation symmetry and its $\mathbb{Z}_{3}$-symmetric equivalents.

4. Do $V-N_{M}$ additional Metropolis sweeps, again at randomly chosen lattice sites.

\section{Critical exponents for the antiferromagnetic $S U(3)$ model}

For the model (5.1) with $\lambda_{4}=0$ we observe a second order transition between symmetric and antiferromagnetic phase. Critical exponents $v$ and $\gamma$ may be introduced in terms of the relations

$$
\chi\left(\lambda_{1, \text { crit }}\right) \propto N^{\gamma / v},\left.\quad \frac{\partial U\left(N, \lambda_{1}\right)}{\partial \lambda_{1}}\right|_{\lambda_{1}=\lambda_{1, \text { crit }}} \propto N^{1 / v} \quad \text { with } \quad U=1-\frac{\left\langle M^{4}\right\rangle}{3\left\langle M^{2}\right\rangle^{2}}, \quad \chi=N^{3}\left\langle M^{2}\right\rangle .
$$

A Monte-Carlo simulation with our modified Wolff cluster algorithm leads to the following critical exponents in comparison to the $\mathbb{Z}_{3}$ Potts values: 


\begin{tabular}{lcc}
\hline \hline exponent & $\mathbb{Z}_{3}$ Potts [8] & minimal Polyakov \\
\hline$v$ & $0.664(4)$ & $0.68(2)$ \\
$\gamma / v$ & $1.973(9)$ & $1.96(2)$ \\
\hline \hline
\end{tabular}

As the exponents coincide (up to statistical errors) the $S U(3)$ model is indeed in the same universality class as the $\mathbb{Z}_{3}$ Potts model (the $X Y$ universality class).

\section{Inverse Monte-Carlo - the basics}

The inverse Monte-Carlo (IMC) method as designed in [9] allows to determine (effective) actions from given configurations. In our case, these are Polyakov loops obtained from gauge configurations generated with the Wilson action. Via IMC we want to determine the couplings of truncated effective actions which (ideally) would give rise to the same distribution of Polyakov loop configurations.

The IMC procedure is based on an ansatz for the effective action of the type $S_{\text {eff }}=\sum_{i} \lambda_{i} S_{i}$. Translational invariance of the reduced Haar measure leads to Schwinger-Dyson equations (SDE), see below. They constitute an overdetermined linear system for the effective couplings $\lambda_{i}$ which may be solved by least-square methods. As a further technical input we require a suitable normalization procedure to make sure that individual equations are appropriately weighted [10].

\section{Geometric SDE from invariant group integrals}

Translational invariance of the Haar measure implies that

$$
\int d \mu_{\mathrm{Haar}}(g)\left(L_{a} f\right)(g)=0 \quad \text { for } f \in L_{2}(G)
$$

with $L_{a}$ being the left derivative on the group. Choosing $f=F L^{a} \chi_{p}$ with a class function $F$ and a fundamental character $\chi_{p}[11]$ one obtains

$$
L_{a}\left(F L^{a} \chi_{p}\right)=F \boldsymbol{L}^{2} \chi_{p}+\left(L_{a} F\right)\left(L^{a} \chi_{p}\right)
$$

and (9.1) reduces to

$$
0=\int d \mu_{\text {Haar }}\left(F \boldsymbol{L}^{2} \chi_{p}+\sum_{q}\left(L_{a} \chi_{p}\right) \frac{\partial F}{\partial \chi_{q}}\left(L^{a} \chi_{q}\right)\right) .
$$

Making use of $\boldsymbol{L}^{2} \chi_{\mu}=-c_{\mu} \chi_{\mu}$ and of

$$
\left(L_{a} \chi_{\mu}\right)\left(L^{a} \chi_{v}\right)=\frac{1}{2}\left(c_{\mu}+c_{v}\right) \chi_{\mu} \chi_{v}-\frac{1}{2} \sum_{\lambda} C_{\mu \nu}^{\lambda} c_{\lambda} \chi_{\lambda}
$$

with Casimir values $c_{\mu}$ and Clebsch-Gordan coefficients $C_{\mu \nu}^{\lambda}$ the equation can be specialized to the case of $S U(3)$. For a suitable function $F$, the SDE finally become

$$
\begin{aligned}
0= & \left\langle-\frac{16}{3} \mathscr{P}_{z} S_{i, \mathscr{P}_{x}}+\left(4 \mathscr{P}_{z}^{*}-\frac{4}{3} \mathscr{P}_{z}^{2}\right) S_{i, \mathscr{P}_{x}, \mathscr{P}_{z}}+\left(6-\frac{2}{3}\left|\mathscr{P}_{z}\right|^{2}\right) S_{i, \mathscr{P}_{x}, \mathscr{P}_{z}^{*}}\right\rangle \\
& -\sum_{j} \lambda_{j}\left\langle\left(4 \mathscr{P}_{z}^{*}-\frac{4}{3} \mathscr{P}_{z}^{2}\right) S_{i, \mathscr{P}_{x}} S_{j, \mathscr{P}_{z}}+\left(6-\frac{2}{3}\left|\mathscr{P}_{z}\right|^{2}\right) S_{i, \mathscr{P}_{x}} S_{j, \mathscr{P}_{z}^{*}}\right\rangle .
\end{aligned}
$$




\section{Algebraic SDE}

For $S U(3)$ (generalizations for $S U(N)$ are possible [11]) we have the identity

$$
\int_{\Omega} d \mathscr{P} d \mathscr{P}^{*} \partial_{\mathscr{P}} f=0
$$

which holds for any function $f$ vanishing on $\partial \Omega$. Choosing

$$
f\left(\mathscr{P}, \mathscr{P}^{*}\right)=J^{3} g\left(\mathscr{P}, \mathscr{P}^{*}\right), \quad g_{x}=\frac{\partial h}{\partial \mathscr{P}_{x}^{*}} \exp (-S), \quad h=S_{i}
$$

we obtain the 'algebraic SDE'

$$
0=\left\langle\frac{3}{2} \frac{\partial J_{z}^{2}}{\partial \mathscr{P}_{z}} S_{i, \mathscr{P}_{x}^{*}}+J_{z}^{2} S_{i, \mathscr{P}_{x}^{*}, \mathscr{P}_{z}}\right\rangle-\sum_{j} \lambda_{j}\left\langle J_{z}^{2} S_{i, \mathscr{P}_{x}^{*}} S_{j, \mathscr{P}_{z}}\right\rangle
$$

\section{IMC results}

We have simulated the underlying YM theory with heat bath methods on different lattice sizes near the critical coupling. The corresponding couplings for the effective models were then determined via IMC [10]. The IMC codes were checked by simulating effective theories with given input couplings which were consistently reproduced by the IMC procedure. In these tests the algebraic and geometric Ward identities led to comparable results, limited only by the statistical accuracy.

We compared the Polyakov loop arising from simulations of full YM theory and from

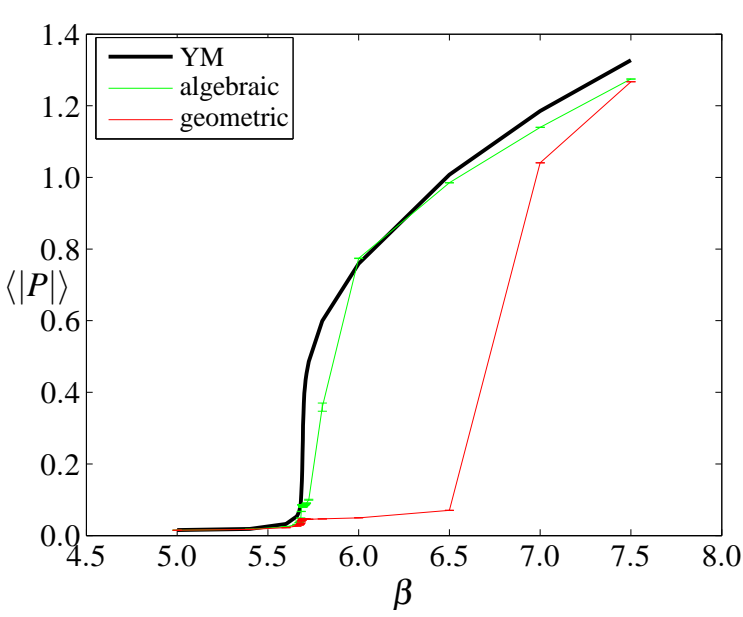

Figure 2: Results obtained from algebraic and geometric SD equations compared to YM results. effective actions based on both geometric and algebraic SDE (on a $16^{3} \times 4$-lattice). We found that the algebraic identities outperformed the geometric ones in reproducing the YM critical behavior, in particular the critical coupling (Fig. 2).

Simulations with algebraic SDE on a $16^{3} \times 4$-lattice allowed to determine up to 11 effective couplings as displayed in Fig. 3 (left panel). The dominant terms in the effective actions are

$$
S_{1}=\sum_{\langle\boldsymbol{x} y\rangle}\left(\chi_{10}\left(\mathscr{P}_{\boldsymbol{x}}\right) \chi_{01}\left(\mathscr{P}_{\boldsymbol{y}}\right)+\text { c.c. }\right), \quad S_{3}=\sum_{\langle\boldsymbol{x} \boldsymbol{y}\rangle} \chi_{11}\left(\mathscr{P}_{\boldsymbol{x}}\right) \chi_{11}\left(\mathscr{P}_{\boldsymbol{y}}\right), \quad S_{5}=\sum_{\boldsymbol{x}} \chi_{11}\left(\mathscr{P}_{\boldsymbol{x}}\right),
$$

i.e. two NN hopping terms and one single-site ('potential') term.

Finally we have extended the IMC procedure to deal with $\mathrm{NN}$ and next-to-NN terms up to order $\mathscr{O}\left(\beta^{3 N_{\mathrm{t}}}\right)$ in the strong coupling expansion. This results in an unstable behavior in the rendering of observables which may be traced to the discontinuities associated with the first order phase transition (Fig. 3, right panel). 

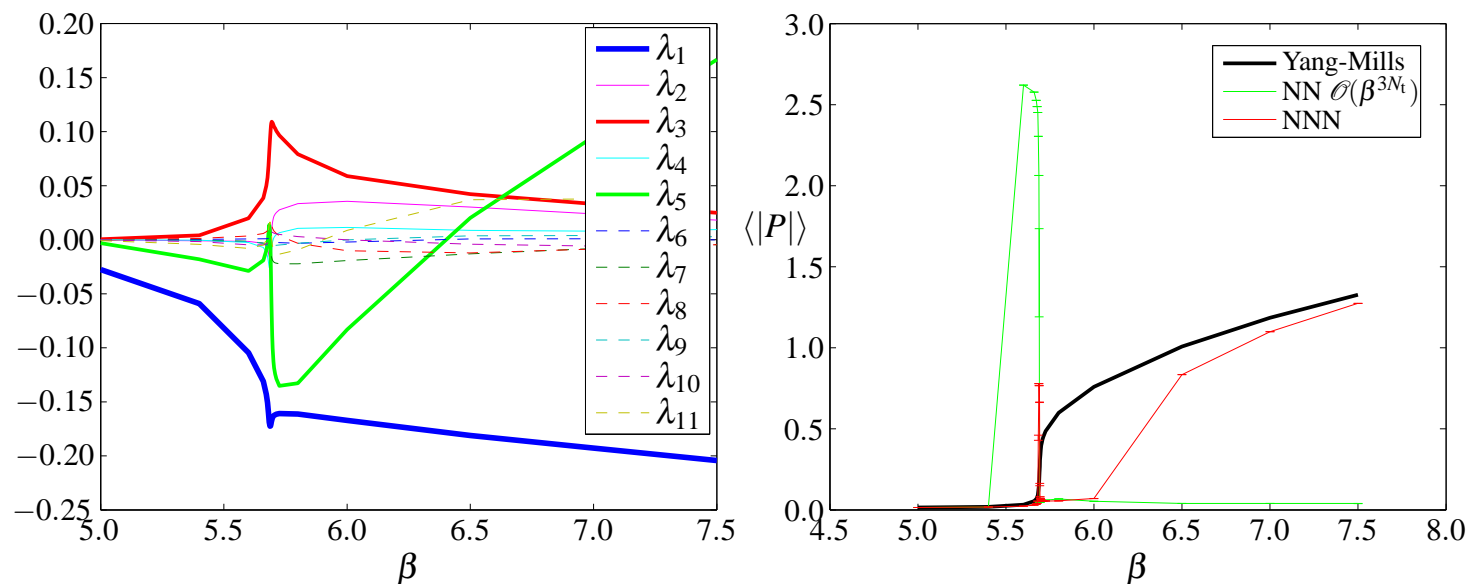

Figure 3: Couplings obtained for $\mathrm{NN}$ interactions up to $\mathscr{O}\left(\beta^{3 N_{\mathrm{t}}}\right)$ (left) and comparison of higher order (next-to-NN) effective theories (right).

\section{Conclusions}

$S U$ (3) Polyakov loop models have a surprisingly rich phase structure when the effective couplings are allowed to vary unrestrictedly. Upon comparing critical exponents for the second-order antiferromagnetic phase transition we have seen that the $S U$ (3) Polyakov loop model is in the same universality class as the $\mathbb{Z}_{3}$ Potts model. The near-perfect agreement between mean-field and MonteCarlo results is due to the fact that the model has a $d=3$ tricritical point. Matching the Polyakov loop models to $S U$ (3) YM theory via IMC leads to stable results only for small lattices and a low number of couplings. Relaxing these restrictions leads to instabilities obscuring, in particular, the location of critical couplings. This behavior is due to the first order nature of the phase transition in lattice gluodynamics. Results for $S U$ (2) YM theory [12], on the other hand, show that IMC is applicable for systems with second order transitions and leads to stable results.

\section{Acknowledgments}

TK acknowledges support by the Konrad-Adenauer-Stiftung e.V. and CW by the Studienstiftung des deutschen Volkes. This work has been supported by the DFG grant Wi 777/8-2.

\section{References}

[1] B. Svetitsky and L. G. Yaffe, Nucl. Phys. B 210, 423 (1982).

[2] R. Falcone, R. Fiore, M. Gravina and A. Papa, arXiv:0704.3882 [hep-lat].

[3] C. Wozar, T. Kaestner, A. Wipf, T. Heinzl and B. Pozsgay, Phys. Rev. D 74 (2006) 114501.

[4] A. Wipf, T. Kaestner, C. Wozar and T. Heinzl, SIGMA 3 (2007) 006.

[5] J. C. Myers and M. C. Ogilvie, arXiv:0707.1869 [hep-lat].

[6] B. A. Berg, Fields Inst. Commun. 26, 1 (2000).

[7] U. Wolff, Phys. Rev. Lett. 62, 361 (1989).

[8] A. P. Gottlob and M. Hasenbusch, Physica A 210, 217 (1994).

[9] M. Falcioni, G. Martinelli, M. L. Paciello, G. Parisi and B. Taglienti, Nucl. Phys. B 265, 187 (1986).

[10] C. Wozar, T. Kaestner, A. Wipf and T. Heinzl, arXiv:0704.2570 [hep-lat].

[11] S. Uhlmann, R. Meinel and A. Wipf, J. Phys. A 40 (2007) 4367.

[12] T. Heinzl, T. Kaestner and A. Wipf, Phys. Rev. D 72 (2005) 065005. 